\title{
A Note on Invariant Submanifolds of Trans-Sasakian Manifolds
}

\author{
Chaogui Hu and Yaning Wang* \\ (Communicated by Levent Kula)
}

\begin{abstract}
The object of this paper is to obtain some necessary and sufficient conditions for an invariant submanifold of a trans-Sasakian manifold to be totally geodesic. In addition, we construct an example to verify our main results.
\end{abstract}

Keywords: invariant submanifold; trans-Sasakian manifold; totally geodesic.

AMS Subject Classification (2010): Primary: 53C15, 53C25; Secondary: 53C40.

\section{Introduction}

The theory of invariant submanifolds of an almost contact manifold has been an interesting area of research in differential geometry for a long time. See, for example, Kon in [11] proved that invariant submanifolds of a Sasakian manifold are totally geodesic if their second fundamental forms are covariantly constant. Kobayashi in [10] proved that a submanifold $M$ of a Kenmotsu manifold with $\xi$ tangent to $M$ is totally geodesic if and only if the fundamental form of $M$ is covariantly constant. In 2009, Sular and Özgür [19] obtained some equivalent conditions regarding some submanifolds of a Kenmotsu manifold being totally geodesic.

In 1985, Oubina [16] introduced a new class of almost contact manifolds, namely, trans-Sasakian manifold of type $(\alpha, \beta)$, which can be considered as a generalization of Sasakian, Kenmotsu and cosymplectic manifolds. Later, several studies (see [4, 20]) have been done on invariant submanifolds of trans-Sasakian manifolds. In 2013, De in [5] obtained that an invariant submanifold of a trans-Sasakian submanifold is totally geodesic if and only if its second fundamental form is 2-pseudo-parallel. Mangione [14] also studied invariant submanifolds of Kenmotsu manifolds. Recently, De and Majhi [6] proved that an invariant submanifold $M$ of a Kenmotsu manifold is totally geodesic if and only if $Q(h, R)=0$ or $Q(S, h)=0$, where $h, R$ and $S$ denote the second fundamental form, curvature tensor and Ricci tensor of $M$, respectively.

Motivated by these results mentioned above, in this paper, we investigate invariant submanifolds of a transSasakian manifold satisfying $Q(S, \bar{\nabla} h)=0, Q(S, \bar{R} \cdot h)=0, Q(g, \bar{R} \cdot h)=0, Q(g, C \cdot h)=0$ or $Q(S, C \cdot h)=0$, respectively, where $C$ and $\bar{R}$ denote the concircular curvature tensor and curvature tensor of $\bar{M}$, respectively.

The present paper is organized as follows. Some preliminaries used to prove our main results are given in Section 2. In Section 3, we obtain some new equivalent conditions for an invariant submanifold of a transSasakian manifold to be totally geodesic. After giving some conclusions of this paper, finally, we construct an example to verify our results in Section 4.

\section{Preliminaries}

Let $\bar{M}$ be a $(2 n+1)$-dimensional smooth differentiable manifold endowed with an almost contact metric structure $(\phi, \xi, \eta, g)$, where $\phi$ is a $(1,1)$-tensor field, $\xi$ a vector field, $\eta$ a one-form and $g$ a compatible Riemannian metric on $\bar{M}$. That is,

$$
\begin{gathered}
\phi^{2} X=-X+\eta(X) \xi, \quad \eta(\xi)=1, \quad \eta(X)=g(X, \xi), \\
g(\phi X, \phi Y)=g(X, Y)-\eta(X) \eta(Y),
\end{gathered}
$$




$$
\phi \xi=0, \quad \eta \circ \phi=0, \quad g(X, \phi Y)=-g(\phi X, Y)
$$

for any $X, Y \in \Gamma(T M)$, where $\Gamma(T M)$ denotes the Lie algebra of all vector fields on $\bar{M}$.

A connected manifold $\bar{M}$ endowed with an almost contact metric structure $(\phi, \xi, \eta, g)$ is called a transSasakian manifold (see [16]) if $(\bar{M} \times R, J, G)$ belongs to the class $W_{4}$ (see [9]), where $J$ is an almost complex structure on $\bar{M} \times R$ which is defined by

$$
J\left(X, f \frac{d}{d t}\right)=\left(\phi X-f \xi, \eta(X) \frac{d}{d t}\right)
$$

for any vector field $X$ on $\bar{M}$ and a smooth function $f$ on $\bar{M} \times R$, and $G$ is the usual product metric on $\bar{M} \times R$. According to [3], an almost contact metric manifold is a trans-Sasakian manifold if and only if

$$
\left(\bar{\nabla}_{X} \phi\right) Y=\alpha\{g(X, Y) \xi-\eta(Y) X\}+\beta\{g(\phi X, Y) \xi-\eta(Y) \phi X\}
$$

for smooth functions $\alpha$ and $\beta$ on $\bar{M}$, where $\bar{\nabla}$ denotes the covariant differentiation with respect to $g$. Generally, $\bar{M}$ is said to be a trans-Sasakian manifold of type $(\alpha, \beta)$. Form equation $(2.4)$, it follows that

$$
\left(\bar{\nabla}_{X} \phi\right) \xi=-\alpha \phi X+\beta(X-\eta(X) \xi), \forall X \in \Gamma(T M) .
$$

Let $M$ be a submanifold of a $(2 n+1)$-dimensional contact metric manifold $\bar{M}$. We denote by $\nabla$ and $\bar{\nabla}$ the Levi-Civita connections of $M$ and $\bar{M}$, respectively. Then for any vector fields $X, Y \in \Gamma(T M)$, the second fundamental form $h$ is given by

$$
\bar{\nabla}_{X} Y=\nabla_{X} Y+h(X, Y) .
$$

Furthermore, for any section $N$ of normal bundle $T^{\perp} M$ we have

$$
\bar{\nabla}_{X} N=-A_{N} X+\nabla \frac{1}{X} N,
$$

where $\nabla^{\perp}$ denotes the normal bundle connection of $M$. The second fundamental form $h$ and shape operator $A_{N}$ are related by

$$
g\left(A_{N} X, Y\right)=g(h(X, Y), N) .
$$

A submanifold $M$ is said to be totally geodesic if $h=0$, which means that the geodesics in $M$ are also geodesics in $\bar{M}$. A submanifold $M$ is said to be semi-parallel (see [7]) (resp. 2-semi-parallel, see [1]) if

$$
\bar{R}(X, Y) \cdot h=0(\text { resp. } \bar{R}(X, Y) \cdot \bar{\nabla} h=0), \forall X, Y \in \Gamma(T M) .
$$

On a Riemannian manifold $M$, for a $(0, k)$-type tensor field $T(k \geq 1)$ and a $(0,2)$-type tensor field $E$, we denote by $Q(E, T)$ a $(0, k+2)$-type tensor field (see [21]), defined as follows

$$
\begin{aligned}
Q(E, T)\left(X_{1}, X_{2}, \cdots, X_{k} ; X, Y\right)= & -T\left(\left(X \wedge_{E} Y\right) X_{1}, X_{2}, \cdots, X_{k}\right) \\
& -T\left(X_{1},\left(X \wedge_{E} Y\right) X_{2}, \cdots, X_{k}\right) \\
& -\cdots-T\left(X_{1}, X_{2}, \cdots, X_{k-1},\left(X \wedge_{E} Y\right) X_{k}\right),
\end{aligned}
$$

where $\left(X \wedge_{E} Y\right) Z=E(Y, Z) X-E(X, Z) Y$.

Moreover, a submanifold $M$ is said to be pseudo-parallel (see [2]) if

$$
\bar{R}(X, Y) \cdot h=f Q(g, h)
$$

holds for any vector fields $X$ and $Y$ tangent to $M$ and a smooth function $f$. Similarly, a submanifold $M$ is said to be 2-pseudo-parallel if $\bar{R}(X, Y) \cdot \bar{\nabla} h=f Q(S, \bar{\nabla} h)$ and Ricci generalized pseudo-parallel (see [15]) if $\bar{R}(X, Y) \cdot h=f Q(S, h)$ for any $X, Y \in \Gamma(T M)$, respectively.

\section{Invariant submanifolds of trans-Sasakian manifolds}

In this section, we shall study an $m$-dimensional submanifold $M^{m}$ of a trans-Sasakian manifold $\bar{M}$ such that the characteristic vector field $\xi$ is tangential to $M$. Generally, $M$ is said to be invariant if $\phi(T M) \subset T M$. On an invariant submanifold $M$ of $\bar{M}$, it follows that $\xi \in \Gamma(T M)$. Note that an invariant submanifold of trans-Sasakian manifolds is also trans-Sasakian (see [19]). From equation (2.5) we obtain directly the following lemma: 
Lemma 3.1 ([18]). Let $M^{m}$ be an invariant submanifold of a trans-Sasakian manifold $\bar{M}$. Then the following equations hold

$$
\nabla_{X} \xi=-\alpha \phi X+\beta(X-\eta(X) \xi), \quad h(X, \xi)=0
$$

for any $X \in \Gamma(T M)$.

Using the above lemma, we obtain the following

Lemma 3.2. Let $M^{m}$ be an invariant submanifold of a trans-Sasakian manifold $\bar{M}$, then we have

$$
\begin{gathered}
R(\xi, X) \xi=\left(\alpha^{2}-\beta^{2}-\xi(\beta)\right)(\eta(X) \xi-X), \\
S(\xi, \xi)=(m-1)\left(\alpha^{2}-\beta^{2}-\xi(\beta)\right)
\end{gathered}
$$

for any $X \in \Gamma(T M)$.

Theorem 3.1. An invariant submanifold of trans-Sasakian manifolds is totally geodesic if and only if $Q(S, \bar{\nabla} h)=0$, provided $\left(\alpha^{2}+\beta^{2}\right)\left(\alpha^{2}-\beta^{2}-\xi(\beta)\right) \neq 0$.

Proof. We assume that $Q(S, \bar{\nabla} h)=0$, this implies

$$
0=Q\left(S, \bar{\nabla}_{X} h\right)(W, K ; U, V)
$$

for any vector fields $X, W, K, U, V \in \Gamma(T M)$. By the above equation and (2.9), we obtain

$$
\begin{aligned}
0= & -\left(\bar{\nabla}_{X} h\right)(S(V, W) U, K)+\left(\bar{\nabla}_{X} h\right)(S(U, W) V, K) \\
& -\left(\bar{\nabla}_{X} h\right)(W, S(V, K) U)+\left(\bar{\nabla}_{X} h\right)(W, S(U, K) V) .
\end{aligned}
$$

Hence, a simple calculation gives that

$$
\begin{aligned}
0= & -\nabla_{X}^{\frac{1}{X}} h(S(V, W) U, K)+h\left(\nabla_{X} S(V, W) U, K\right)+h\left(S(V, W) U, \nabla_{X} K\right) \\
& +\nabla_{X}^{\perp} h(S(U, W) V, K)-h\left(\nabla_{X} S(U, W) V, K\right)-h\left(S(U, W) V, \nabla_{X} K\right) \\
& -\nabla_{X}^{\perp} h(W, S(V, K) U)+h\left(\nabla_{X} W, S(V, K) U\right)+h\left(W, \nabla_{X} S(V, K) U\right) \\
& +\nabla_{X}^{\perp} h(W, S(U, K) V)-h\left(\nabla_{X} W, S(U, K) V\right)-h\left(W, \nabla_{X} S(U, K) V\right)
\end{aligned}
$$

for any vector fields $X, W, K, U, V \in \Gamma(T M)$. Putting $K=W=\xi$ in the above equation and using Lemma 3.1, we obtain

$$
0=S(V, \xi) h\left(U, \nabla_{X} \xi\right)-S(U, \xi) h\left(V, \nabla_{X} \xi\right) .
$$

In particularly, substituting $V=\xi$ in (3.4) we obtain

$$
S(\xi, \xi) h\left(U, \nabla_{X} \xi\right)=0 .
$$

On a trans-Sasakian manifold, we have (see [5])

$$
h(X, \phi U)=\phi h(X, U) .
$$

From Lemma 3.1, (3.5) and (3.6), we obtain

$$
\alpha S(\xi, \xi) \phi h(U, X)=\beta S(\xi, \xi) h(U, X) .
$$

Applying $\phi$ on both sides of equation (3.7), we obtain

$$
-\alpha S(\xi, \xi) h(U, X)=\beta S(\xi, \xi) \phi h(U, X) .
$$

By Lemma 3.2, (3.7) and (3.8), we obtain

$$
(m-1)\left(\alpha^{2}+\beta^{2}\right)\left(\alpha^{2}-\beta^{2}-\xi(\beta)\right) h(U, X)=0,
$$

for any vector fields $X$ and $U \in \Gamma(T M)$. If $m=1$, that is, $T M=\operatorname{Span}\{\xi\}$, then $M$ is anti-invariant (see [13]). Therefore, it follows that an invariant submanifold of trans-Sasakian manifolds with $Q(S, \bar{\nabla} h)=0$ is totally geodesic provided that $\left(\alpha^{2}+\beta^{2}\right)\left(\alpha^{2}-\beta^{2}-\xi(\beta)\right) \neq 0$. The converse follows trivially. 
Corollary 3.1. An invariant submanifold of Sasakian manifolds is totally geodesic if and only if $Q(S, \bar{\nabla} h)=0$.

Corollary 3.2. An invariant submanifold of Kenmotsu manifolds is totally geodesic if and only if $Q(S, \bar{\nabla} h)=0$.

Theorem 3.2. An invariant submanifold of trans-Sasakian manifolds is totally geodesic if and only if $Q(S, \bar{R} \cdot h)=0$, provided $\alpha^{2}-\beta^{2}-\xi(\beta) \neq 0$.

Proof. Let us consider $Q(S, \bar{R} \cdot h)=0$, then it follows that

$$
0=Q(S, \bar{R}(X, Y) \cdot h)(W, K ; U, V)
$$

for any vector fields $X, Y, W, K, U, V \in \Gamma(T M)$. We obtain directly from the above equation and (2.9) that

$$
\begin{aligned}
0= & -(\bar{R}(X, Y) \cdot h)(S(V, W) U, K)+(\bar{R}(X, Y) \cdot h)(S(U, W) V, K) \\
& -(\bar{R}(X, Y) \cdot h)(W, S(V, K) U)+(\bar{R}(X, Y) \cdot h)(W, S(U, K) V) .
\end{aligned}
$$

By the definition of $\bar{R} \cdot h$, we obtain

$$
\begin{aligned}
0= & -S(V, W)\left[R^{\perp}(X, Y) h(U, K)-h(R(X, Y) U, K)-h(R(X, Y) K, U)\right] \\
& +S(U, W)\left[R^{\perp}(X, Y) h(V, K)-h(R(X, Y) V, K)-h(R(X, Y) K, V)\right] \\
& -S(V, K)\left[R^{\perp}(X, Y) h(W, U)-h(R(X, Y) W, U)-h(R(X, Y) U, W)\right] \\
& \left.+S(U, K)\left[R^{\perp}(X, Y) h(W, V)-h(R(X, Y) W, V)-h(R(X, Y) V), W\right)\right] .
\end{aligned}
$$

Putting $Y=W=K=V=\xi$ in the above equation, we obtain immediately

$$
S(\xi, \xi) h(R(X, \xi) \xi, U)=0
$$

Form Lemma 3.2 and relation (3.9), we obtain $(m-1)\left(\alpha^{2}-\beta^{2}-\xi(\beta)\right)^{2} h(X, U)=0$ for any vector fields $X, U \in \Gamma(T M)$. Proceeding similarly as in proof of Theorem 3.1, we complete the proof.

Corollary 3.3. An invariant submanifold of Sasakian manifolds is totally geodesic if and only if $Q(S, \bar{R} \cdot h)=0$.

Corollary 3.4. An invariant submanifold of Kenmotsu manifolds is totally geodesic if and only if $Q(S, \bar{R} \cdot h)=0$.

Theorem 3.3. An invariant submanifold of trans-Sasakian manifolds is totally geodesic if and only if $Q(g, \bar{R} \cdot h)=0$, provided $\alpha^{2}-\beta^{2}-\xi(\beta) \neq 0$.

Proof. Considering $Q(g, \bar{R} \cdot h)=0$, this is equivalent to

$$
0=Q(g, \bar{R}(X, Y) \cdot h)(W, K ; U, V)
$$

for any $X, Y, W, K, U, V \in \Gamma(T M)$. The following equation follows directly from (2.9) and the above equation

$$
\begin{aligned}
0= & -(\bar{R}(X, Y) \cdot h)(g(V, W) U, K)+(\bar{R}(X, Y) \cdot h)(g(U, W) V, K) \\
& -(\bar{R}(X, Y) \cdot h)(W, g(V, K) U)+(\bar{R}(X, Y) \cdot h)(W, g(U, K) V) .
\end{aligned}
$$

By the definition of $\bar{R} \cdot h$, we obtain

$$
\begin{aligned}
0= & -g(V, W)\left[R^{\perp}(X, Y) h(U, K)-h(R(X, Y) U, K)-h(U, R(X, Y) K]\right. \\
& +g(U, W)\left[R^{\perp}(X, Y) h(V, K)-h(R(X, Y) V, K)-h(V, R(X, Y) K)\right] \\
& -g(V, K)\left[R^{\perp}(X, Y) h(W, U)-h(R(X, Y) W, U)-h(W, R(X, Y) U)\right] \\
& +g(U, K)\left[R^{\perp}(X, Y) h(W, V)-h(R(X, Y) W, V)-h(W, R(X, Y) V)\right] .
\end{aligned}
$$

In particularly, putting $Y=W=K=V=\xi$ in the above equation and applying Lemma 3.1 we obtain

$$
h(U, R(X, \xi) \xi)=0
$$

By Lemma 3.2 and (3.10), we have $\left(\alpha^{2}-\beta^{2}-\xi(\beta)\right) h(U, X)=0$ for any vector fields $X, U \in \Gamma(T M)$. This completes the proof. 
Corollary 3.5. An invariant submanifold of Sasakian manifolds is totally geodesic if and only if $Q(g, \bar{R} \cdot h)=0$.

Corollary 3.6. An invariant submanifold of Kenmotsu manifolds is totally geodesic if and only if $Q(g, \bar{R} \cdot h)=0$. [5])

For a $(2 n+1)$-dimensional Riemannian manifold $\bar{M}$, the concircular curvature tensor $C$ is defined by (see

$$
C(X, Y) Z=\bar{R}(X, Y) Z-\frac{\tau}{2 n(2 n+1)}[g(Y, Z) X-g(X, Z) Y]
$$

for any vector fields $X, Y, Z \in \Gamma(T M)$, where $\tau$ is the scalar curvature of $M$. We also have

$$
(C(X, Y) \cdot h)(U, V)=R^{\perp}(X, Y) h(U, V)-h(C(X, Y) U, V)-h(U, C(X, Y) V) .
$$

Theorem 3.4. An invariant submanifold of trans-Sasakian manifolds is totally geodesic if and only if $Q(g, C \cdot h)=0$, provided $\tau \neq 2 n(2 n+1)\left(\alpha^{2}-\beta^{2}-\xi(\beta)\right)$.

Proof. Assuming that $Q(g, C \cdot h)=0$, then we get

$$
0=Q(g, C(X, Y) \cdot h)(W, K ; U, V)
$$

for any vector fields $X, Y, W, K, U, V \in \Gamma(T M)$. From (2.9), (3.12) and the above equation we obtain

$$
\begin{aligned}
0= & -g(V, W)(C(X, Y) \cdot h)(U, K)+g(U, W)(C(X, Y) \cdot h)(V, K) \\
& -g(V, K)(C(X, Y) \cdot h)(W, U)+g(U, K)(C(X, Y) \cdot h)(W, V) \\
= & -g(V, W)\left[R^{\perp}(X, Y) h(U, K)-h(C(X, Y) U, K)-h(C(X, Y) K, U)\right] \\
& +g(U, W)\left[R^{\perp}(X, Y) h(V, K)-h(C(X, Y) V, K)-h(C(X, Y) K, V)\right] \\
& -g(V, K)\left[R^{\perp}(X, Y) h(W, U)-h(C(X, Y) W, U)-h(C(X, Y) U, W)\right] \\
& +g(U, K)\left[R^{\perp}(X, Y) h(W, V)-h(C(X, Y) W, V)-h(C(X, Y) V, W)\right] .
\end{aligned}
$$

In particularly, by Lemma 3.1 , we put $Y=K=W=U=\xi$ in the above equation and obtain

$$
h(C(X, \xi) \xi, V)=0 .
$$

By Lemma 3.2 and (3.11), we obtain immediately

$$
C(X, \xi) \xi=\left(\alpha^{2}-\beta^{2}-\xi(\beta)-\frac{\tau}{2 n(2 n+1)}\right)(X-\eta(X) \xi) .
$$

Substituting (3.14) into (3.13) yields that

$$
\left(\alpha^{2}-\beta^{2}-\xi(\beta)-\frac{\tau}{2 n(2 n+1)}\right) h(X, V)=0
$$

for any $X, V \in \Gamma(T M)$. This completes the proof.

Corollary 3.7. An invariant submanifold of Sasakian manifolds is totally geodesic if and only if $Q(g, C \cdot h)=0$, provided $\tau \neq 2 n(2 n+1)$.

Corollary 3.8. An invariant submanifold of Kenmotsu manifolds is totally geodesic if and only if $Q(g, C \cdot h)=0$, provided $\tau \neq-2 n(2 n+1)$.

Theorem 3.5. An invariant submanifold of trans-Sasakian manifolds is totally geodesic if and only if $Q(S, C \cdot h)=0$, provided $\left(\alpha^{2}-\beta^{2}-\xi(\beta)\right)\left[\tau-2 n(2 n+1)\left(\alpha^{2}-\beta^{2}-\xi(\beta)\right)\right] \neq 0$.

Proof. For any $X, Y, U, V, W, K \in \Gamma(T M)$, we see that

$$
0=Q(S, C(X, Y) \cdot h)(W, K ; U, V) .
$$


We obtain the following equation by (2.9), (3.12) and the above equation

$$
\begin{aligned}
0= & -S(V, W)(C(X, Y) \cdot h)(U, K)+S(U, W)(C(X, Y) \cdot h)(V, K) \\
& -S(V, K)(C(X, Y) \cdot h)(W, U)+S(U, K)(C(X, Y) \cdot h)(W, V) \\
= & -S(V, W)\left[R^{\perp}(X, Y) h(U, K)-h(C(X, Y) U, K)-h(C(X, Y) K, U)\right] \\
& +S(U, W)\left[R^{\perp}(X, Y) h(V, K)-h(C(X, Y) V, K)-h(C(X, Y) K, V)\right] \\
& -S(V, K)\left[R^{\perp}(X, Y) h(W, U)-h(C(X, Y) W, U)-h(C(X, Y) U, W)\right] \\
& +S(U, K)\left[R^{\perp}(X, Y) h(W, V)-h(C(X, Y) W, V)-h(C(X, Y) V, W)\right] .
\end{aligned}
$$

In particularly, using Lemma 3.1 and putting $Y=K=W=U=\xi$ in the above equation we have

$$
S(\xi, \xi) h(C(X, \xi) \xi, V)=0 .
$$

By Lemma 3.2, (3.14) and (3.15), we obtain

$$
\left(\alpha^{2}-\beta^{2}-\xi(\beta)\right)\left[\left(\alpha^{2}-\beta^{2}-\xi(\beta)\right)-\frac{\tau}{2 n(2 n+1)}\right] h(X, V)=0
$$

for any $X, V \in \Gamma(T M)$. This completes the proof.

Corollary 3.9. An invariant submanifold of Sasakian manifolds is totally geodesic if and only if $Q(S, C \cdot h)=0$, provided $\tau \neq 2 n(2 n+1)$.

Corollary 3.10. An invariant submanifold of Kenmotsu manifolds is totally geodesic if and only if $Q(S, C \cdot h)=0$, provided $\tau \neq-2 n(2 n+1)$.

For an invariant submanifold $M$ of a trans-Sasakian manifold with $\alpha$ and $\beta$ being constants, from our main results, [5] and [18] we see that the following conditions are equivalent:

- $M$ is totally geodesic,

- the second fundamental form of $M$ is parallel with $\alpha^{2}+\beta^{2} \neq 0$,

- the second fundamental form of $M$ is semi-parallel with $\alpha^{2}+\beta^{2} \neq 0$,

- the second fundamental form of $M$ is recurrent with $\alpha^{2}+\beta^{2} \neq 0$,

- the second fundamental form of $M$ is 2-recurrent with $\alpha^{2}+\beta^{2} \neq 0$,

- the second fundamental form of $M$ is 2-semi-parallel with $\alpha^{2}+\beta^{2} \neq 0$,

- the second fundamental form of $M$ is pseudo-parallel with either $\alpha^{2}-\beta^{2}-f \neq 0$ or $\alpha \beta \neq 0$,

- the second fundamental form of $M$ is 2-pseudo-parallel with $\alpha^{2}+\beta^{2} \neq 0$,

- the second fundamental form of $M$ is Ricci generalized pseudo-parallel with either $\left(\alpha^{2}-\beta^{2}\right)(2 n f-1) \neq$ 0 or $\alpha \beta \neq 0$,

- the second fundamental form of $M$ satisfies $C \cdot h=0$ with either $\alpha^{2}-\beta^{2} \neq \frac{\tau}{2 n(2 n+1)}$ or $\alpha \beta \neq 0$,

- the second fundamental form of $M$ satisfies $Q(S, \bar{\nabla} h)=0$ and $\alpha^{2} \neq \beta^{2}$,

- the second fundamental form of $M$ satisfies $Q(S, \bar{R} \cdot h)=0$ and $\alpha^{2} \neq \beta^{2}$,

- the second fundamental form of $M$ satisfies $Q(g, \bar{R} \cdot h)=0$ and $\alpha^{2} \neq \beta^{2}$,

- the second fundamental form of $M$ satisfies $Q(g, C \cdot h)=0$ and $\tau \neq 2 n(2 n+1)\left(\alpha^{2}-\beta^{2}\right)$,

- the second fundamental form of $M$ satisfies $Q(S, C \cdot h)=0$ and $\alpha^{2} \neq \beta^{2}$ and $\tau \neq 2 n(2 n+1)\left(\alpha^{2}-\beta^{2}\right) \neq 0$.

\section{Example}

We consider a five-dimensional manifold $\widetilde{M}=\left\{\left(x_{1}, x_{2}, x_{3}, x_{4}, t\right) \in R^{5}: t \neq 0\right\}$, where $\left(x_{1}, x_{2}, x_{3}, x_{4}, t\right)$ are standard coordinates in $R^{5}$. We choose the vector fields

$$
e_{1}=e^{-t} \frac{\partial}{\partial x_{1}}, \quad e_{2}=e^{-t} \frac{\partial}{\partial x_{2}}, \quad e_{3}=e^{-t} \frac{\partial}{\partial x_{3}}, e_{4}=e^{-t} \frac{\partial}{\partial x_{4}}, e_{5}=e^{-t} \frac{\partial}{\partial t},
$$

which are linearly independent at each point of $\widetilde{M}$. We define $g$ by

$$
g=e^{2 t} K
$$


where $K$ is the Euclidean metric on $R^{5}$. Hence $\left\{e_{1}, e_{2}, e_{3}, e_{4}, e_{5}\right\}$ is an orthonormal basis of $\widetilde{M}$.

We consider an 1-form $\eta$ defined by

$$
\eta=e^{t} d t, \eta(X)=g\left(X, e_{5}\right), \quad \forall X \in T \widetilde{M} .
$$

We define the $(1,1)$ tensor field $\phi$ by

$$
\phi\left\{\sum_{i=1}^{2}\left(x_{i} \frac{\partial}{\partial x_{i}}+x_{i+2} \frac{\partial}{\partial x_{i+2}}+t \frac{\partial}{\partial t}\right)\right\}=\sum_{i=1}^{2}\left(x_{i} \frac{\partial}{\partial x_{i+2}}-x_{i+2} \frac{\partial}{\partial x_{i}}\right) .
$$

Thus, we have

$$
\phi\left(e_{1}\right)=e_{3}, \phi\left(e_{2}\right)=e_{4}, \phi\left(e_{3}\right)=-e_{1}, \phi\left(e_{4}\right)=-e_{2}, \quad \phi\left(e_{5}\right)=0 .
$$

The linear property of $g$ and $\phi$ yields that

$$
\begin{aligned}
& \eta\left(e_{5}\right)=1, \quad \phi^{2}(X)=-X+\eta(X) e_{5} \\
& g(\phi X, \phi Y)=g(X, Y)-\eta(X) \eta(Y),
\end{aligned}
$$

for any vector fields $X, Y$ on $\widetilde{M}$. Thus, $\widetilde{M}(\phi, \xi, \eta, g)$ defines an almost contact metric manifold with $e_{5}=\xi$. Moreover, let $\widetilde{\nabla}$ be the Levi-Civita connection with respect to metric $g$. Then we have

$$
\begin{aligned}
{\left[e_{1}, e_{2}\right] } & =e^{-t} \frac{\partial}{\partial x_{1}}\left(e^{-t} \frac{\partial}{\partial x_{2}}\right)-e^{-t} \frac{\partial}{\partial x_{2}}\left(e^{-t} \frac{\partial}{\partial x_{1}}\right) \\
& =e^{-2 t} \frac{\partial^{2}}{\partial x_{1} \partial x_{2}}-e^{-2 t} \frac{\partial^{2}}{\partial x_{2} \partial x_{1}} \\
& =0 .
\end{aligned}
$$

Similarly,

$$
\begin{gathered}
{\left[e_{1}, \xi\right]=e^{-t} e_{1}, \quad\left[e_{2}, \xi\right]=e^{-t} e_{2}, \quad\left[e_{3}, \xi\right]=e^{-t} e_{3},} \\
{\left[e_{4}, \xi\right]=e^{-t} e_{4}, \quad\left[e_{i}, e_{j}\right]=0, \quad 1 \leq i \neq j \leq 4 .}
\end{gathered}
$$

The Riemannian connection $\widetilde{\nabla}$ of the metric $g$ is given by

$$
\begin{aligned}
2 g\left(\widetilde{\nabla}_{X} Y, Z\right)= & X g(Y, Z)+Y g(Z, X)-Z g(X, Y)-g(X,[Y, Z]) \\
& -g(Y,[X, Z])+g(Z,[X, Y]),
\end{aligned}
$$

By Koszul's formula, we obtain the following equations

$$
\begin{gathered}
\widetilde{\nabla}_{e_{1}} e_{1}=-e^{-t} \xi, \quad \widetilde{\nabla}_{e_{2}} e_{2}=-e^{-t} \xi, \quad \widetilde{\nabla}_{e_{3}} e_{3}=-e^{-t} \xi, \quad \widetilde{\nabla}_{e_{4}} e_{4}=-e^{-t} \xi, \\
\widetilde{\nabla}_{\xi} \xi=0, \quad \widetilde{\nabla}_{\xi} e_{i}=0, \quad \widetilde{\nabla}_{e_{i}} \xi=e^{-t} e_{i}, \quad 1 \leq i \leq 4
\end{gathered}
$$

and $\widetilde{\nabla}_{e_{i}} e_{j}=0$ for all $1 \leq i, j \leq 4$. Thus, we see that $M$ is a trans-Sasakian manifold of type $\left(0, e^{-t}\right)$.

Let $M$ be a subset of $\widetilde{M}$ and consider the isometric immersion $f: M \mapsto \widetilde{M}$ defined by

$$
f\left(x_{1}, x_{3}, t\right)=\left(x_{1}, 0, x_{3}, 0, t\right) .
$$

It can be easily proved that $M=\left\{\left(x_{1}, x_{3}, t\right) \in R^{3}:\left(x_{1}, x_{3}, t\right) \neq 0\right\}$ is a 3-dimensional submanifold of $\widetilde{M}$, where $\left(x_{1}, x_{3}, t\right)$ are standard coordinates in $R^{3}$. We choose the vector fields

$$
e_{1}=e^{-t} \frac{\partial}{\partial x_{1}}, \quad e_{3}=e^{-t} \frac{\partial}{\partial x_{3}}, \quad e_{5}=e^{-t} \frac{\partial}{\partial t},
$$

which are linearly independent at each point of $M$. We define $g_{1}$ by

$$
g_{1}=e^{2 t} K_{1},
$$


where $K_{1}$ is the Euclidean metric on $R^{3}$. Hence $\left\{e_{1}, e_{3}, e_{5}\right\}$ is an orthonormal basis of $M$. We define 1 -form $\eta_{1}$ and $(1,1)$-form tensor field $\phi_{1}$ respectively by

$$
\eta_{1}=e^{t} d t, \quad \eta_{1}(X)=g_{1}\left(X, e_{5}\right), \quad \forall X \in \Gamma(T M)
$$

and

$$
\phi_{1}\left\{\left(x_{1} \frac{\partial}{\partial x_{1}}+x_{3} \frac{\partial}{\partial x_{3}}+t \frac{\partial}{\partial t}\right)\right\}=\left(x_{1} \frac{\partial}{\partial x_{3}}-x_{3} \frac{\partial}{\partial x_{1}}\right) .
$$

Thus, we have

$$
\phi_{1}\left(e_{1}\right)=e_{3}, \quad \phi_{1}\left(e_{3}\right)=-e_{1}, \quad \phi_{1}\left(e_{5}\right)=0 .
$$

The linear property of $g_{1}$ and $\phi_{1}$ yields that

$$
\begin{aligned}
& \eta_{1}\left(e_{5}\right)=1, \quad \phi_{1}^{2}(X)=-X+\eta_{1}(X) e_{5}, \\
& g_{1}\left(\phi_{1} X, \phi_{1} Y\right)=g_{1}(X, Y)-\eta_{1}(X) \eta_{1}(Y)
\end{aligned}
$$

for any vector fields $X, Y$ on $M$. Thus, $M$ is an invariant submanifold of $\widetilde{M}$ with $e_{5}=\xi$. Let $\nabla$ be the induced connection. Using Koszul's formula, we obtain

$$
\begin{gathered}
\nabla_{e_{1}} e_{1}=-e^{-t} \xi, \quad \nabla_{e_{3}} e_{3}=-e^{-t} \xi, \quad \nabla_{\xi} \xi=0, \quad \nabla_{e_{1}} \xi=e^{-t} e_{1}, \\
\nabla_{e_{3}} \xi=e^{-t} e_{3}, \quad \nabla_{\xi} e_{1}=0, \quad \nabla_{\xi} e_{3}=0, \quad \nabla_{e_{1}} e_{3}=0, \quad \nabla_{e_{3}} e_{1}=0 .
\end{gathered}
$$

Finally, from the values of $\widetilde{\nabla}_{e_{i}} e_{j}$ and $\nabla_{e_{l}} e_{k}$, where $i, j \in\{1,2,3,4,5\}$ and $l, k \in\{1,3,5\}$, we obtain $h=0$ and hence $M$ is totally geodesic. Thus, Theorems $3.1,3.2,3.3,3.4$ and 3.5 are verified.

\section{Acknowledgments}

The second author was supported by the National Natural Science Foundation of China (No. 11526080), Key Scientific Research Program in Universities of Henan Province (No. 16A110004), the Research Foundation for the Doctoral Program of Henan Normal University (No. qd14145) and the Youth Science Foundation of Henan Normal University (No. 2014QK01).

\section{References}

[1] Arslan, K., Lumiste, U., Murathn, C. and Özgür, C., 2-semiparallel surfaces in space forms, I: two particular cases, Proc. Est. Acad. Sci. Phys. Math. 49 (2000), no. 3, 139-148.

[2] Asperti, A. C., Lobos, G. A. and Mercuri, F., Pseudo-parallel immersions in space forms, Mat. Contemp. 17 (1999), 59-70.

[3] Blair, D. E. and Oubina, J. A., Conformal and related changes of metric on the product of two almost contact metric manifolds, Publ. Mat. 34 (1990), no. 1, 199-207.

[4] Chinea, D. and Prestelo, P. S., Invariant submanifolds of a trans-Sasakian manifold, Publ. Math. Debrecen 38 (1991), no, 1-2, 103-109.

[5] De, A., Totally geodesic submanifolds of a trans-Sasakian manifold, Proc. Est. Acad. Sci. 62 (2013), no. 4, 249-257.

[6] De, U. C. and Majhi, P., On invariant submanifolds of Kenmotsu manifolds, J. Geom. 106 (2015), no. 1, 109-122.

[7] Deprez, J., Semi-parallel surfaces in Euclidean space, J. Geom. 25 (1985), no. 2, 192-200.

[8] Deshmukh, S. and Tripathi, M. M., A note on trans-Sasakian manifolds, Math. Slovaca 63 (2013), no. 6, 1361-1370.

[9] Gray, A. and Hervella, L. M., The sixteen classes of almost Heritian manifolds and their linear invariants, Ann. Mat. Pura Appl. 123 (1980), no. $1,35-58$.

[10] Kobayashi, M., Semi-invariant submanifolds of a certain class of almost contact metric manifolds, Tensor (N.S.) 43 (1986), no. 1, $28-36$.

[11] Kon, M., Invariant submanifols of normal contact metric manifolds, Kodai Math. Sem. Rep. 25 (1973), no. 3, 330-336.

[12] Kowalczyk, D., On some subclass of semisymmetric manifolds, Soochow J. Math. 27 (2001), no. 4, 445-462.

[13] Lotta, A., Slant submanifolds in contact geometry, Bull. Math. Soc. Roumanie 39 (1996), no. 1-4, 183-198.

[14] Mangione, V., Totally geodesic submanifolds of a Kenmotsu space form, Math. Reports 7 (2005), no. 4, 315-324.

[15] Murathan, C., Arslan, K. and Ezentas, E., Ricci generalized pseudo-symmetric immersions, Differ. Geom. Appl. 99-108, Matfyzpress, Prague, 2005.

[16] Oubina, J. A., New classes of almost contact metric structures, Publ. Math. Debrecen 32 (1985), no. 3-4, 187-193.

[17] Prasad, R. and Srivastava, V., Some results on trans-Sasakian manifofold, Mat. Vesnik. 65 (2013), no. 3, 346-352.

[18] Sarkar, A. and Sen, M., On invariant submanifolds of trans-Sasakian manifolds, Proc. Est. Acad. Sci. 61 (2012), no. 1, $29-37$.

[19] Sular, S. and Özgür, C., On some submanifolds of Kenmotsu manifolds, Chaos Soliton Fract. 42 (2009), no. 4, 1990-1995.

[20] Vanli, A. T. and Sari, R., Invariant submanifolds of trans-Sasakian manifolds, Differ. Geom. Dyn. Syst. 12 (2010), 277-288.

[21] Verstraelen, L., Comments on pseudosymmetry in the sense of Ryszard Deszcz, Geometry and Topology of submanifolds, 6 (1994), no. 1, 199-209. 


\section{Affiliations}

CHAOGUI Hu

AdDREss: Henan Normal University, School of Mathematics and Information Sciences, Henan Engineering Laboratory for Big Data Statistical Analysis and Optimal Control, 453007, Xinxiang, Henan, P. R. China. E-MAIL: cgh805@163.com

YANING WANG

ADDRESS: Henan Normal University, School of Mathematics and Information Sciences, Henan Engineering Laboratory for Big Data Statistical Analysis and Optimal Control, 453007, Xinxiang, Henan, P. R. China.

E-MAIL: wyn051@163.com 